\title{
Aiming for quality digital health in cancer: Insights from literature and stakeholders informing the Australian Digital Health in Cancer Roadmap.
}

\section{Introduction}

- Digital health approaches promise to improve access and personalisation in cancer care. ${ }^{[1]}$

- Whilst enthusiastically adopted, some have limited efficacy and safety data.

- To inform the Australian Digital Health in Cancer Roadmap, we conducted a literature meta-review and interviewed stakeholders about barriers, enablers, needs and opportunities for quality digital health in cancer.

\section{Methods}

- Systematic literature meta-review (Jan 2013-June 2018),

- Stakeholder consultations (telephone interviews/focus groups) with consumers (14), health care providers (9), researchers (6), NGO representatives (6), policy/government representatives (10), and technology developers (6)

- Thematic analysis by two researchers; coding framework (i) developed from subset of literature review (ii) iteratively developed through subsequent coding (literature \& stakeholder consultations)

\section{Results: literature screening}

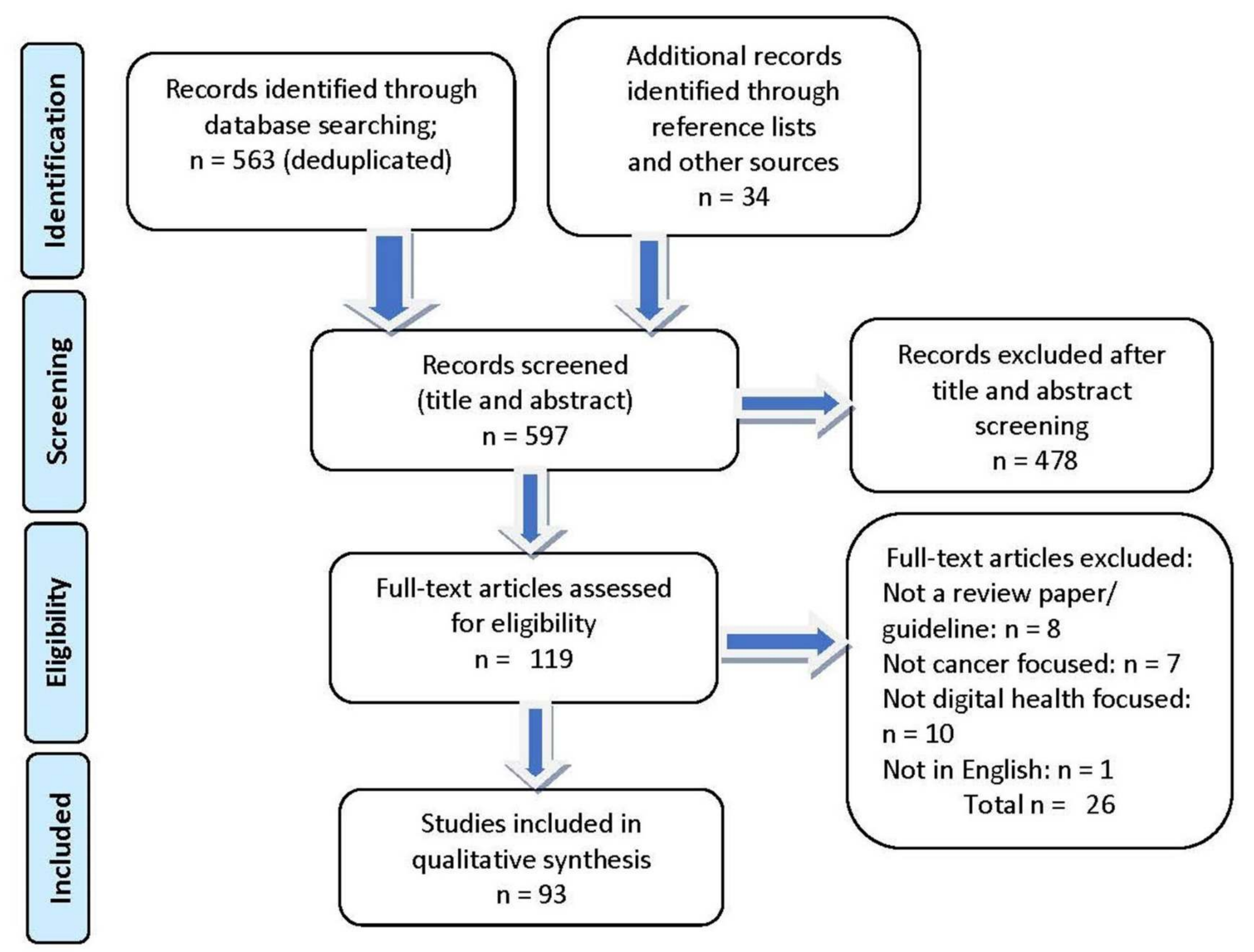

Mean $\operatorname{AMSTAR}^{[2]}$ quality rating $=5.95 / 11$
Bogda Koczwara ${ }^{1,2}$, Emma Kemp ${ }^{1,2}$, Joshua Trigg ${ }^{3}$, Lisa Beatty ${ }^{1,2,3}$, Anthony Maeder ${ }^{1}$, Haryana Dhillon $^{4}$, Vince McCauley ${ }^{5}$, Trish Williams ${ }^{1}$. $\quad$ bogda.koczwara@flinders.edu.au

\section{Results: Themes relating to quality and safety}

-Barriers: Limited evidence overall for usability, acceptability, feasibility, efficacy, effectiveness, cost effectiveness, sustained effects; low grade evidence/low quality trials, lack of standardisation

- Enablers: Evidence for usability, feasibility, efficacy for some technologies/purposes/populations - Needs: More reliable, robust, higher level evidence; standardisation of research methods/measures - Opportunities: Further evaluation in diverse populations/sub-groups

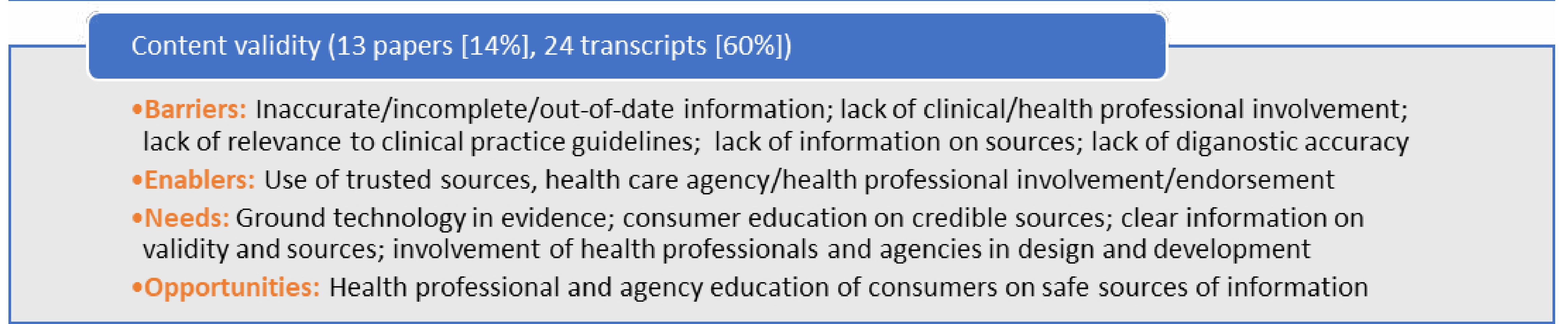

Ease of use (43 papers [46\%], 39 trancripts [98\%])

-Barriers: Lack of user-friendliness; lack of efficiency gains; lack of interoperability with existing electronic

systems; lack of integration with health care site workflow, lack of training/resourcing

- Enablers: Easy access/continuity across locations; efficiency gains; integration/connectivity between

systems/providers; sector interest; clear value; edcuation; workflow integration

- Needs: User-friendly 'intuitive' design; integrated/standardised systems; live, shared electronic medical

record; quality workplace change management including human resources

-Opportunities: improved ease of record keeping, symptom tracking, self-management

\section{Safety and risk (general) (26 papers [28\%], 18 trancripts [45\%])}

-Barriers: Lack of evidence/testing and clinical input; dissemination of inaccurate/unsafe information; risks of unsupervised interventions (e.g. exercise); incorrect/incomplete system use; lack of legal recourse

Enablers: Built-in auditing, checks and balances (electronic systems); existing evidence for reduced error reduced emergency presentations; advice on web and mobile apps to check with health professionals

- Needs: Inbuilt checks and balances, strong evidence for benefit with minimal risk of harm

-Opportunities: Consumer education on safe sources of information; improved auditing capacity

Ethics, privacy, security (23 papers [25\%], 29 transcripts [73\%])
- Barriers: Lack of appropriate privacy/security measures; consumer concerns - data ownership and privacy
- Enablers: Algorithms, structures and processes to protect privacy and security
- Needs: Establish consumer-focused standards for ethics, privacy, security; design, evaluation, and
transparency of evaluation/accreditation according to these standards
-Opportunities: n/a
Governance (13 papers [14\%], 6 transcripts [15\%])
-Barriers: Lack of regulation and evaluation; lack of information on developers; issues with legal compliance
- Enablers: Good local management/governance e.g. of moderation of social media forums
-Needs: Establishment of regulating guidelines, accreditation process; establishment of regulating body;
dissemination of accreditation process such that this is available and clear to consumers
-Opportunities: Use of existing voluntary certificiations (Afgis, HONCode) or health agency endorsement
Coordination (2 papers [2\%], 34 transcripts [85\%])
- Barriers: Lack of coordination/intrgration at health site, state and federal level; lack of political leadership
and investment; siloed/repetitive development; research process outpaced by technology development
-Enablers: Passionate 'champions', dedicated staff, leadership, advocates, cultural change, partnerships
- Needs: Development of overall direction/framework for development, consumer focus (patient centred),
political willpower; collaboration between research bodies, sites, states; sufficent investment
-Opportunities: Collaboration between research bodies to develop and run large high quality trials of co-
developed digital health technologies; one stop shop/portal for disseminating existing technologies.

\section{Conclusions}

Compared with literature, Australian stakeholders place less emphasis on evidence and more emphasis on the need for a coordinated approach responsive to consumer needs.

A strategic framework is needed for digital health in cancer that defines standards, measures, and data collection.

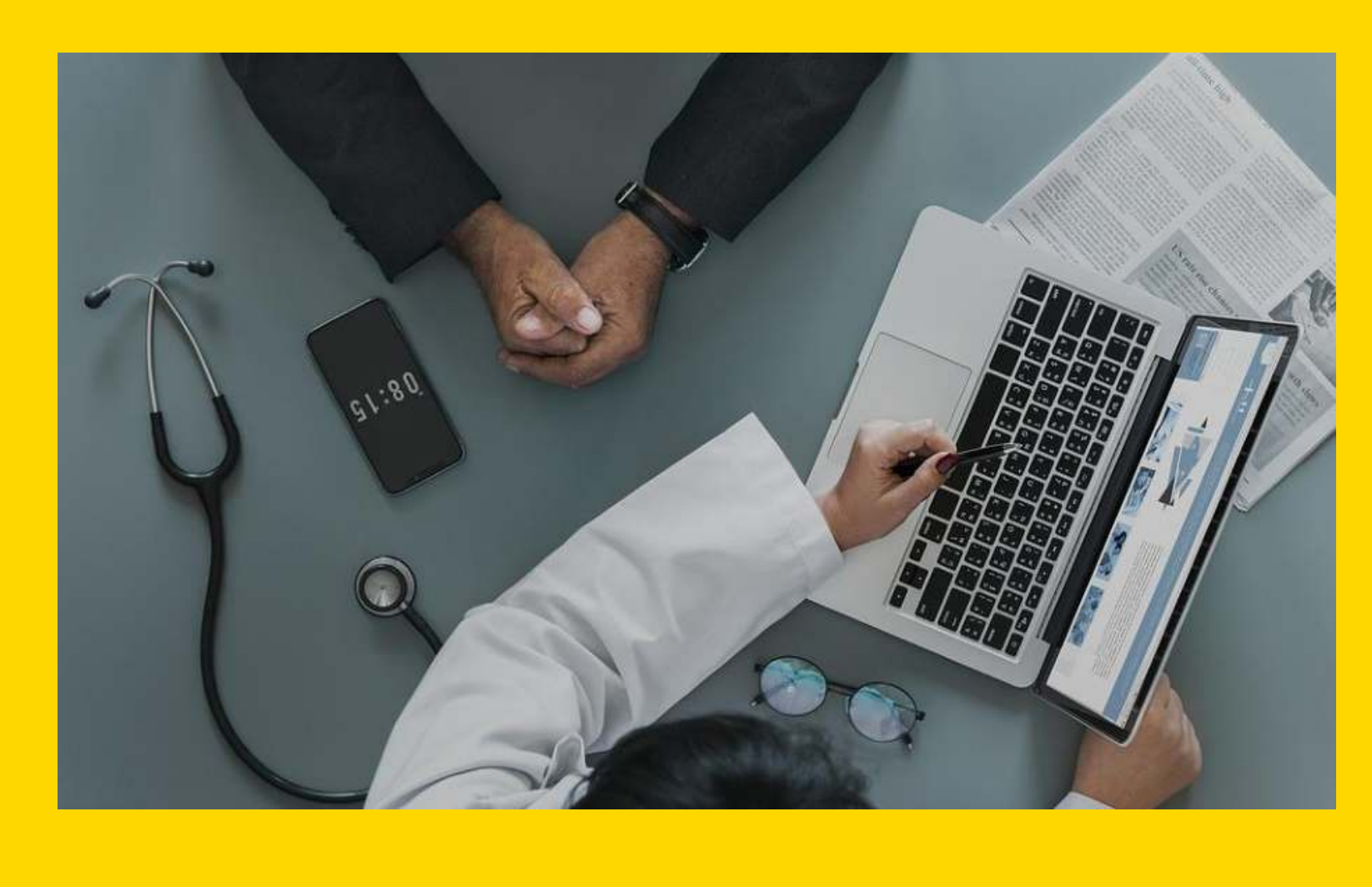

This project was funded by a Flinders University School of Medicine Capacity Building Research Grant. [1] Ventura, Filipa, Joakim Öhlén, and Ingalill Koinberg. "An integrative review of supportive e-health programs in cancer care." European Journal of Oncology Nursing 17.4 (2013): 498-507. [2] Shea, Beverley J., et al. "Development of AMSTAR: a measurement tool to assess the methodological quality of systematic reviews." BMC medical research methodology 7.1 (2007): 10. 